\title{
Twist mode in atomic Fermi gases
}

\author{
X. Vinas, ${ }^{1}$ R. Roth, ${ }^{2}$ P. Schuck, ${ }^{3}$ and J. Wambach ${ }^{4}$ \\ ${ }^{1}$ Departament d'Estructura i Constituents de la Matèria, Facultat de Física, Universitat de Barcelona, Diagonal 647, \\ E-08028 Barcelona, Spain \\ ${ }^{2}$ Gesellschaft für Schwerionenforschung, Planckstrasse 1, D-64291 Darmstadt, Germany \\ ${ }^{3}$ Institut des Sciences Nucléaires, Université Joseph Fourier, CNRS-IN2P3, 53 Avenue des Martyrs, \\ F-38026 Grenoble-Cédex, France \\ ${ }^{4}$ Institut für Kernphysik, Technische Universität Darmstadt, Schlossgartenstrasse 9, D-64289 Darmstadt, Germany
} (Received 21 March 2001; revised manuscript received 22 June 2001; published 3 October 2001)

\begin{abstract}
The quantum-kinetic energy of a finite number of trapped fermionic atoms provides a restoring force for shear motion due to a distortion of the momentum distribution. In analogy to the twist mode of nuclear physics, it is proposed that counter rotating the upper and lower hemisphere of a spherical atomic cloud yields a finite-frequency mode closely related to transverse zero sound waves in bulk Fermi liquids.
\end{abstract}

DOI: 10.1103/PhysRevA.64.055601

PACS number(s): 03.75.Fi, 05.30.Jp

The advent of Bose-Einstein condensation of trapped atomic ${ }^{87} \mathrm{Rb}$ in 1995 has initiated large experimental and theoretical activities in the field of very dilute, almost ideal Bose gases [1,2]. The recent experimental achievements of trapping fermionic alkali atoms [3] raise hope that much progress will also be made in the near future for Fermi gases. Indeed, Fermi-Dirac degeneracy of a mixture of trapped ${ }^{40} \mathrm{~K}$ atoms in two different hyperfine states has been achieved [4]. Recently, also the cooling of a mixture of bosonic ${ }^{7} \mathrm{Li}$ and fermionic ${ }^{6} \mathrm{Li}$ atoms into a quantum degenerate regime was accomplished [5]. On the theoretical side, intensive studies have started as well. For instance, the possibility that certain gas species show attractive interaction (e.g., ${ }^{6} \mathrm{Li}$ ) has initiated studies on the possible superfluidity of such systems $[6,7]$. The close analogy with another finite Fermi system, the nucleus, has been pointed out [7]. It is indeed tempting to transpose many typical features of atomic nuclei to trapped atomic Fermi gases. Besides the very spectacular superfluidity properties, there is interest in the spectrum of collective excitations, most of them showing features proper to Landau's zero sound modes in bulk Fermi liquids [8]. For finite Fermi systems, zero sound translates into modes analogous to those of an elastic body $[9,10]$. One of the most remarkable examples is the so-called "twist mode" [11-13] in spherical nuclei for which there is experimental evidence from backward inelastic electron scattering [14]. This mode has also been predicted to exist in medium-to-heavy spherical alkali metal clusters as the most prominent magnetic multipole excitation [15]. In a macroscopic picture, the twist mode comprises a coherent oscillation of the particles in the upper-half sphere versus those in the lower-half sphere. For small amplitudes, it corresponds to a purely kinetic excitation without any spatial distortion of the equilibrium shape. In this note, we wish to investigate to what extent the twist mode may also occur as a collective mode in very dilute, atomic Fermi gases in the degenerate limit.

It is well known that the twist mode is generated by the operator [12]

$$
T=e^{-i \alpha z l_{z}}=e^{\alpha \vec{u} \cdot \vec{\nabla}}, \quad \vec{u}=(y z,-x z, 0),
$$

acting on the ground-state wave function of the Fermi system. As is evident, $T$ induces a rotation of the particles around the body-fixed $z$ axis with a rotation angle proportional to $z$, i.e., the rotation is clockwise for $z>0$ and counterclockwise for $z<0$. The amplitude of this twist is characterized by the angle $\alpha$. One can verify that the twist corresponds to a magnetic mode of spin-parity $J^{\pi}=2^{-}$. Although the operator (1) induces no change in the spatial distribution, the momenta become locally distorted. The subsequent derivation of the mode frequency will closely follow the original work of Holzwarth and Eckart [12]. For atomic Fermi gases with $N \simeq 10^{5}-10^{6}$ particles, the Thomas-Fermi approach is very appropriate [7] (as is the case of atomic bosons [16]). Most relevant for our purpose is the total kinetic energy of the system

$$
E_{k i n}(\alpha)=\int \frac{d^{3} r d^{3} p}{(2 \pi \hbar)^{3}} \frac{p^{2}}{2 m} f_{\alpha}(\vec{r}, \vec{p}),
$$

where $f_{\alpha}$ is the distorted phase-space distribution in the Thomas-Fermi approximation

$$
f_{\alpha}=\nu \theta\left[\tilde{p}_{F}^{2}(\vec{r}, \hat{p})-p^{2}\right] .
$$

Here, $\nu$ is the degeneracy factor and $\theta$ the unit step function. The (quadrupole) deformed local momentum is given by

$$
\begin{aligned}
\tilde{p}_{F}(\vec{r}, \hat{p})= & p_{F}(\vec{r}) N(\alpha)\left\{1-\alpha \sqrt{\frac{2 \pi}{15}}\left[y\left(Y_{21}(\hat{p})-Y_{2-1}(\hat{p})\right)\right.\right. \\
& \left.\left.+i x\left(Y_{21}(\hat{p})+Y_{2-1}(\hat{p})\right)\right]\right\}
\end{aligned}
$$

where

$$
p_{F}(\vec{r})=\sqrt{2 m\left[\mu-V_{e x}(\vec{r})-g(\nu-1) \rho(\vec{r})\right]}
$$

denotes the local Fermi momentum with chemical potential $\mu$ and trapping potential $V_{e x}(\vec{r})$ and 
TABLE I. The twist-mode frequencies $\Omega_{T}$ in units of the trap frequency $\omega$ for several numbers of ${ }^{6} \mathrm{Li}$ $(g<0)$ and ${ }^{40} \mathrm{~K}(g>0)$ atoms in each hyperfine state for different scattering lengths (in units of the Bohr radius). The frequencies of the harmonic-oscillator traps are $\omega=2 \pi \times 144 \mathrm{~Hz}$ for ${ }^{6} \mathrm{Li}$ and $\omega=2 \pi$ $\times 33.5 \mathrm{~Hz}$ for ${ }^{40} \mathrm{~K}$, which corresponds to level spacings of $\hbar \omega=6.9$ and $1.6 \mathrm{nK}$, respectively.

\begin{tabular}{lcccccc}
\hline \hline \multicolumn{1}{c}{$N$} & $a$ (units of $\left.a_{0}\right)$ & $\Omega_{T}($ units of $\omega)$ & $a\left[a_{0}\right]$ & $\Omega_{T}[\omega]$ & $a\left[a_{0}\right]$ & $\Omega_{T}[\omega]$ \\
\hline${ }^{6} \mathrm{Li}$ & & & & & & \\
$1 \times 10^{3}$ & -2063.0 & 1.042 & -206.3 & 1.004 & -20.63 & 1.000 \\
$5 \times 10^{3}$ & -2063.0 & 1.056 & -206.3 & 1.005 & -20.63 & 1.000 \\
$1 \times 10^{4}$ & -2063.0 & 1.064 & -206.3 & 1.006 & -20.63 & 1.001 \\
$5 \times 10^{4}$ & -2063.0 & 1.087 & -206.3 & 1.007 & -20.63 & 1.001 \\
$1 \times 10^{5}$ & -2063.0 & 1.101 & -206.3 & 1.008 & -20.53 & 1.001 \\
$2 \times 10^{5}$ & -2063.0 & 1.116 & -206.3 & 1.009 & -20.63 & 1.001 \\
$5 \times 10^{5}$ & -2063.0 & 1.142 & -206.3 & 1.011 & -20.63 & 1.001 \\
${ }^{40} \mathrm{~K}$ & & & & & & \\
$1 \times 10^{3}$ & 157.0 & 0.996 & 15.7 & 1.000 & 1570.0 & 0.966 \\
$1 \times 10^{4}$ & 157.0 & 0.995 & 15.7 & 0.999 & 1570.0 & 0.952 \\
$1 \times 10^{5}$ & 157.0 & 0.992 & 15.7 & 0.999 & 1570.0 & 0.932 \\
$1 \times 10^{6}$ & 157.0 & 0.989 & 15.7 & 0.999 & 1570.0 & 0.906 \\
$1 \times 10^{7}$ & 157.0 & 0.984 & 15.7 & 0.998 & 1570.0 & 0.873 \\
\hline \hline
\end{tabular}

$$
N(\alpha)=1-\alpha^{2} \frac{x^{2}+y^{2}}{15}
$$

In the dilute gas limit, it can be assumed that the two-body interaction is given by its long-wavelength limit

$$
v\left(\vec{r}-\vec{r}^{\prime}\right)=g \delta\left(\vec{r}-\vec{r}^{\prime}\right),
$$

with $g=4 \pi \hbar^{2} a / m$, where $a$ is the $s$-wave scattering length. For trapped atomic fermions two situations can occur. For example, ${ }^{40} \mathrm{~K}$ can be trapped as a mixture of atoms in two different $m_{F}$ states, $m_{F}=9 / 2$ and $m_{F}=7 / 2$ [4]. In this case, $s$-wave scattering is realized and the interaction (7) is active. In contrast, if the atoms are all in a single hyperfine state, there can be no $s$-wave scattering. In the latter case, $p$-wave interactions may become very important [17]. The inclusion of $p$-wave interactions, as well as the description of FermionBoson mixtures, requires a substantial extension of the fluiddynamical formalism and will be discussed in a following publication. In the present report, we concentrate on a twocomponent Fermi gas with an equal number of particles in each magnetic substate. In this case, the equilibrium Thomas-Fermi equation for the density $\rho(\vec{r})$ of atoms in one of the two $m_{F}$ states is given by $[7,17]$

$$
\rho(\vec{r})=\frac{p_{F}^{3}(\vec{r})}{6 \pi^{2} \hbar^{3}},
$$

which leads to a cubic equation for $\rho$ that can be solved analytically. To second order in $\alpha$, we then obtain

$$
E_{k i n}(\alpha)=\int d^{3} r \tau_{0}(\vec{r})\left[1+\frac{\alpha^{2}}{3}\left(x^{2}+y^{2}\right)\right]
$$

where $\tau_{0}$ is the total kinetic-energy density at equilibrium

$$
\tau_{0}(\vec{r})=2 \frac{3}{5} \frac{\hbar^{2}}{2 m} \frac{p_{F}^{5}(\vec{r})}{6 \pi^{2} \hbar^{3}}
$$

Since in lowest order, the potential energy contains no contribution from the twist mode, we obtain for the restoring force (assuming a spherically symmetric trap of harmonic oscillator shape with frequency $\omega$ )

$$
C=\frac{\partial^{2} E_{k i n}(\alpha)}{\partial \alpha^{2}}=\frac{16 \pi}{9} \int d r r^{4} \tau_{0}(r) .
$$

We also need to evaluate the mass parameter $B$ of the twist motion. As usual, in fluid dynamics it is given by

$$
\begin{aligned}
B & =m \int d^{3} r 2 \rho(\vec{r}) u^{2} \\
& =m \int d^{3} r 2 \rho(\vec{r}) z^{2}\left(x^{2}+y^{2}\right) \\
& =\frac{16 \pi}{15} m \int d r r^{6} \rho(r),
\end{aligned}
$$

where $2 \rho(\vec{r})$ corresponds to the total density of atoms. The twist frequency $\Omega_{T}$ is then obtained as

$$
\hbar \Omega_{T}=\sqrt{\frac{C}{B}} .
$$

We have considered two systems. One is ${ }^{6} \mathrm{Li}$ with a very large attractive scattering length of $a=-2063 a_{0} \quad\left(a_{0}\right.$ $=$ Bohr radius) [18]. The trapping potential was taken to be $\hbar \omega=6.9 \mathrm{nK}[5]$. The other system is ${ }^{40} \mathrm{~K}$ with a repulsive scattering length of $a=157 a_{0}$ and $\hbar \omega=1.6 \mathrm{nK}$ [19]. The results for the twist frequency as a function of the particle number in each magnetic substate are given in Table I. In 
order to see how $\Omega_{T}$ depends on the interaction strength (which may vary due to the tuning of Feshbach resonances) we also list $\Omega_{T}$ for various other values of the scattering lengths (differing from the original ones by powers of 10).

From Table I, it can be inferred that the influence of the interaction on the twist frequency is very moderate. It is typically of the order of $10 \%$ (except for the very large particle number). This is consistent with the expectation that, for transverse zero sound, $s$-wave interactions give no contribution to the restoring force [13]. The dependence of the twist frequency on the interaction only enters through the mass parameter $B$ that depends on the density [Eq. (12)]. Depending on the sign of the interaction, the gas either expands (repulsive) or contracts (attractive) relative to the free gas case. Consequently, the frequency is decreased (increased) with respect to its noninteracting value $\Omega_{T 0}=\omega$ for repulsion (attraction). This feature should be measurable even though the absolute effect might be small.

Very interesting possibilities arise when the $p$-wave interaction becomes strong [17]. In this case, there will be a significant correction to the kinetic energy through the effective mass, and hence, a large influence on the twist frequency. One may even encounter instabilities, signaled by the exponential growth of the twist amplitude. Also, as mentioned above, one can then have a direct influence of the interaction in a one component Fermi system. Another interesting issue is how the twist mode is influenced by eventual superfluidity. It can be predicted that the twist mode ceases to exist once pairing is strong enough for the system to reach its irrotational flow limit [7]. There may, however, be intermediate situations. To our knowledge, these possibilities have not been addressed for the nuclear twist.

The question how to excite the twist mode in the experiment may not be trivial. One could imagine utilizing the well-developed technique of rotating trapped atoms [20]. First, a very elongated trap potential is created. Subsequently, a rotating laser field is wrapped around the long axis inducing a rotation of the atomic cloud. If, instead of applying the laser field parallel to the long axis, it is incident at a certain angle $\phi$ and a mirror is placed parallel to the long axis that reflects the laser beam in such a way that it hits the other hemisphere at an angle $-\phi$, then the first hemisphere will rotate in one direction and the other hemisphere in the opposite direction (at least approximately) since the rotational sense of the laser is inverted by the mirror. If the rotation is very gentle and stopped at a certain time, the system will continue oscillating (approximately) in the twist mode. Whether one can detect the twist mode by switching off the trap potential and subsequently imaging the velocity distribution of the expanding atoms remains an open question.

The above-mentioned experimental possibility for exciting the twist mode came up in a discussion with Y. Castin, J. Dalibard, and C. Salomon and is gratefully acknowledged. We also thank A. Richter for fruitful discussions on the nuclear twist mode. One of us (X.V) acknowledges financial support from DGCYT (Spain) under Grant No. PB98-1247 and from the DGR (Catalonia) under Grant No. 2000SGR00024.
[1] A.S. Parkins and D.F. Walls, Phys. Rep. 303, 1 (1998).

[2] F. Dalfovo, S. Giorgini, L.P. Pitaevskii, and S. Stringari, Rev. Mod. Phys. 71, 463 (1999).

[3] E.R.I. Abraham, W.I. McAlexander, C.A. Sackett, and R.G. Hulet, Phys. Rev. Lett. 74, 1315 (1995); F.S. Cataliotti, E.A. Cornell, C. Fort, M. Iguscio, F. Marin, M. Prevedelli, L. Ricci, and G.M. Tino, Phys. Rev. A 57, 1136 (1998).

[4] B. DeMarco and D.S. Jin, Science 285, 1703 (1999).

[5] A.G. Truscott, K.E. Strecker, W.I. McAlexander, G.B. Partridge, and R.G. Hulet, Science 291, 2570 (2001).

[6] M. Houbiers, R. Ferwerda, H.T.C. Stoof, W.I. McAlexander, C.A. Sackett, and R.G. Hulet, Phys. Rev. A 56, 4864 (1997).

[7] M. Farine, P. Schuck, and X. Viñas, Phys. Rev. A 62, 013608 (2000).

[8] G. Baym and C. Pethick, The Physics of Liquid and Solid He (Wiley, New York, 1978), Part II, Chap. 1.

[9] H. Lamb, Proc. London Math. Soc. 13, 189 (1882).

[10] G.F. Bertsch, Ann. Phys. (Leipzig) 86, 138 (1974); G.F. Bertsch and R.A. Broglia, Oscillations in Finite Quantum Sys- tems (Cambridge University Press, Cambridge, England, 1994).

[11] G. Holzwarth and G. Eckart, Z. Phys. A 283, 219 (1977).

[12] G. Holzwarth and G. Eckart, Nucl. Phys. A 325, 1 (1979).

[13] B. Schwesinger, K. Pingel, and G. Holzwarth, Nucl. Phys. A 341, 1 (1980); B. Schwesinger, Phys. Rev. C 29, 1475 (1984).

[14] P. von Neumann-Cosel et al., Phys. Rev. Lett. 82, 1105 (1999).

[15] V.O. Nesterenko, J.R. Marinelli, F.F. de Souza Cruz, W. Kleinig, and P.G. Reinhard, Phys. Rev. Lett. 85, 3141 (2000).

[16] P. Schuck and X. Viñas, Phys. Rev. A 61, 043603 (2000).

[17] R. Roth and H. Feldmeier, J. Phys. B 33, L787 (2000); R. Roth and H. Feldmeier, e-print cond-mat/0102416, to be published in Phys. Rev. A (2001).

[18] E.R.I. Abraham, W.I. McAlexander, J.M. Gerton, R.G. Hulet, R. Côtéand, and A. Dalgarno, Phys. Rev. A 55, R3299 (1997).

[19] B. DeMarco, J.L. Bohn, J.P. Burke, Jr, M. Holland, and D.S. Jin, Phys. Rev. Lett. 82, 4208 (1999).

[20] K.W. Madison, F. Chevy, W. Wohlleben, and D. Dalibard, Phys. Rev. Lett. 84, 806 (2000). 